\title{
SOBRE LA JUSTIFICACIÓN DE LA DEMOCRACIA EN LA OBRA DE CARLOS S. NINO
}

i expresiones como «los jueces crean Derecho» acostumbran a suscitar profundas controversias $^{1}$, otras como «legitimidad de la democracia» parecen caracterizarse precisamente por la virtud opuesta: la democracia se considera justificada desde perspectivas filosófico-políticas que van de la llamada «teoría elitista de la democracia» al socialismo democrático. No obstante, si examinamos de cerca los postulados teóricos de estas perspectivas, encontramos desacuerdos genuinos sobre el tipo de democracia del que cabe predicar la nota de la legitimidad así como sobre el alcance de dicha legitimidad. Así, podemos considerar que la coincidencia de estas teorías al afirmar la «legitimidad de la democracia» es producto de un pseudoacuerdo que encubre un desacuerdo genuino.

El objeto de estas páginas no es otro que el de tratar de aclarar algunos aspectos de la teoría sobre la «legitimidad de la democracia» elaborada por Carlos S. Nino. Examinaré, en primer lugar, a qué tipo de «democracia» se refiere la teoría de Nino -la democracia como modelo ideal de carácter procesal- para, a continuación, preguntarme por la clase de «legitimidad» que cabe predicar de dicho modelo, respondiendo a esta cuestión con lo que me ha parecido la reconstrucción más plausible de la teoría de Nino sobre los tipos de justicia procesal: la democracia queda legitimada como un caso de «justicia procesal instrumental imperfecta». Finalmente, formularé alguna conclusión en torno a los límites de este tipo de justificación de la democracia.

1. La teoría de la democracia de Carlos S. Nino no constituye una reflexión filosófico-política en torno a las concretas realidades político-institucionales de la democracia, sino que, por el contrario, se trata más bien de un intento de fundamentación de la democracia pp. 68 y ss.

${ }^{1}$ Genaro R. Carrió: Notas sobre Derecho y lenguaje. Abeledo-Perreot, Buenos Aires, 1973, 
a partir de un modelo ideal de carácter procesal. Para comprender la anterior afirmación hay que aludir a dos distinciones relevantes en los diversos discursos en los que aparece el término «democracia»: la distinción entre democracia real e ideal, por un lado, y entre democracia formal y material, por otro.

1.1 La oposición entre democracia real y democracia ideal hace referencia a dos denotaciones diferentes del término «democracia»: Por un lado, alude a los sistemas políticos que usualmente considerados como «democráticos»y, por otro, a la democracia entendida como «modelo ideal». Situarse en el primer plano permite desarrollar tanto una teoría descriptiva como propuestas normativas acerca de los sistemas políticos democráticos existentes, mientras que, desde el segundo plano, únicamente tiene sentido la construcción de una teoría normativa.

Como acabo de apuntar, Nino se sitúa en el segundo plano, es decir, opta por una teoría normativa de la democracia basada en un modelo ideal:

«La democracia es un "sucedáneo" del discurso moral;... se trata de una especie de discurso moral "regimentado" que preserva en más alto grado que cualquier otro sistema de decisiones los rasgos del discurso moral originario, pero apartándose de exigencias que hacen que este discurso sea un método inestable e inconcluyente para arribar a decisiones colectivas» ${ }^{2}$.

Lo que desde esta teoría se trata de justificar no es el sistema político democrático entendido como realidad histórica e institucional, sino el procedimiento democrático de toma de decisiones en unas condiciones fuertemente idealizadas. Estamos en presencia de un discurso que para nada entra a considerar si en el mundo real se producen o no desviaciones a la hora de tomar decisiones supuestamente democráticas.

Pese a este distanciamiento del mundo real, una teoría normativa como ésta, inspirada en un modelo ideal, no tiene por qué ser un ejercicio argumentativo inútil sino que, en principio, puede cumplir dos finalidades: Por un lado, dotar de justificación derivada a las democracias reales, en la medida en que éstas recojan los imperativos del modelo ideal. $\mathrm{Y}$, por otro lado, desarrollar una función regulativa y crítica, al servir como medida

${ }^{2}$ Carlos S. Nino: Ética y derechos humanos. Un ensayo de fundamentación (2. a edición), Astrea, Buenos Aires, 1989, p. 388. 
de evaluación de las democracias realmente existentes y como modelo al que deben tender.

En mi opinión, la primera función debe relativizarse bastante pues -como veremosla justificación de la democracia real queda fuertemente debilitada al difuminarse o desaparecer la mayoría de los imperativos del modelo ideal esbozados por $\mathrm{Nino}^{3}$. En cambio, la segunda función adquiere una mayor relevancia conforme decae la primera, ya que si bien es cierto que las condiciones de justicia impuestas por tales modelos difícilmente permiten aceptar como justas ninguna de las democracias realmente existentes, también lo es que establecen un horizonte normativo al que las democracias reales deben tender.

Esta visión normativa de la democracia basada en modelos ideales puede expresarse gráficamente como sigue:

1.2 La segunda precisión que el término «democracia» obliga a realizar se

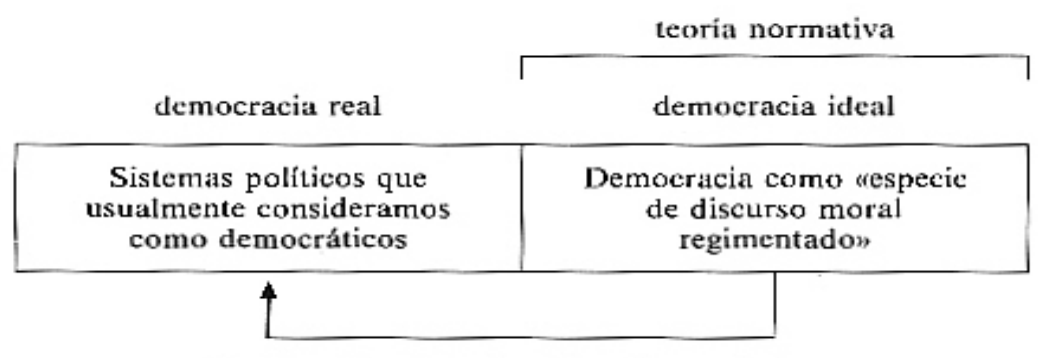

justificación derivada / función regulativa

refiere a la oposición entre democracia formal y democracia material. Como es sabido, la primera noción alude a una serie de formas, procedimientos o reglas del juego ${ }^{4}$, mientras que la democracia material o real hace referencia a una serie de fines o contenidos. En este segundo sentido, la democracia

${ }^{3}$ Tales como los que «debe tratarse que las decisiones estén justificadas sobre la base de principios que satisfagan las condiciones formales del discurso moral, que para ello deben escucharse los argumentos a favor o en contra de que esta satisfacción se dé, que toda persona moral debe considerarse una fuente potencial de tales argumentos, que todas ellas deben participar en las decisiones colectivas, etcétera». En ob. cit., p. 392.

${ }^{4}$ En síntesis, estos requisitos serían los señalados por Bobbio en: Norberto Bobbio (et alter), voz «democracia», Diccionario de política, Siglo XXI Editores, Madrid, 1982, pp. 506-507. 
exige un reparto igualitario de poder, pero no sólo del poder político, sino también del poder económico y social.

Como ya he apuntado, Nino sostiene -con las precisiones que luego haré- una fundamentación procesal de la democracia, pues, a la hora de llevar a cabo una teoría normativa de la misma, se centra en su aspecto formal. Afirmar que la democracia puede fundamentarse como «una especie de discurso moral regimentado» supone admitir que la democracia queda legitimada sin necesidad de entrar a analizar la justicia de sus contenidos. Es más, Nino considera que pueda justificarse incluso en el caso de que se haya incurrido en «desvíos morales» respecto de sus contenidos ${ }^{5}$.

La opción por una teoría de tipo normativo, basada en un modelo ideal de carácter procesal, puede representarse gráficamente como sigue:

2. Una vez que la democracia queda configurada como un modelo ideal de

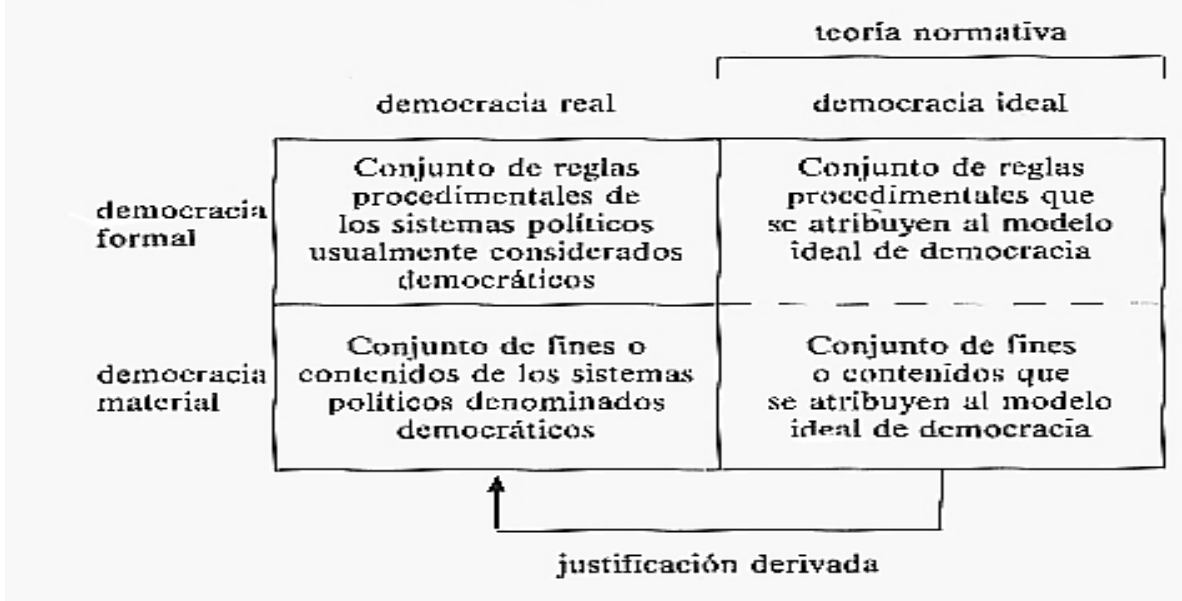

carácter procesal, cabe preguntarse en qué sentido podemos predicar la «legitimidad» de dicho modelo. La respuesta a esta pregunta viene condicionada por el tipo de justicia procesal ante el que nos encontramos. En este sentido

${ }^{5}$ Carlos S. Nino: Ética y derechos humanos. Un ensayo de fundamentación (2. a edición), Astrea, Buenos Aires, 1989, pp. 370-371. 
veremos que, si bien en un primer momento Nino afirma que la democracia constituye un supuesto de «justicia procesal pura imperfecta», esta tesis es luego desplazada por la más débil del «valor epistemológico de la democracia». A partir de aquí, trataré de demostrar que la reconstrucción más plausible de esta última tesis lleva a sostener que la democracia es un caso de «justicia procesal instrumental imperfecta».

2.1. Efectivamente, en la primera edición de su libro Ética y derechos humanos ${ }^{6}$ Nino afirma que la democracia constituye un caso de «justicia procesal pura imperfecta». Dicha fórmula se obtiene partiendo de una clasificación de Rawls entre los distintos tipos de justicia procesal e introduciendo varias modificaciones.

Rawls distingue entre tres tipos de justicia procesal ${ }^{7}$ : la «justicia procesal pura», en la que la justicia de un resultado viene dada solamente por el procedimiento seguido para obtenerlo, sin que haya criterios independientes para juzgar la justicia de ese resultado. La «justicia procesal perfecta», en la que el procedimiento «conduce infaliblemente a resultados considerados justos según criterios independientes». Y la «justicia procesal imperfecta», en la que el procedimiento «tiende a producir, pero no asegura, resultados considerados justos según criterios independientes (como en el caso de un buen procedimiento judicial)».

Nino se resiste a considerar la democracia como un caso de «justicia procesal imperfecta» sin más, ya que esta visión instrumental de la misma «recoge parte, pero no todo, de lo que la hace moralmente superior (lo que recoge es, principalmente, la circunstancia de que la democracia contribuye, mejor que cualquier otro sistema, a expandir el discurso moral propiamente dicho)»» ${ }^{8}$.

Esto le lleva a observar cómo los dos últimos tipos de justicia procesal «son análogos entre sí y se distinguen del primero por el hecho de ser de carácter instrumental», es decir, en ambos casos habría que recurrir a instancias extrañas al procedimiento para poder predicar la justicia de los resultados. Además, ambos tipos se diferencian entre sí únicamente por la mayor o menor fiabilidad del procedimiento. Hay por lo tanto una asimetría en la clasificación: mientras la justicia procesal instrumental es susceptible de dividirse en perfecta e imperfecta, la justicia procesal pura carece de tal subdivisión. Esto obliga a Nino a plantearse si tendría

\footnotetext{
241-243.

${ }^{6}$ Carlos S. Nino: Ética y derechos humanos, Paidos Studio/ Básica, Buenos Aires, 1984, pp.

${ }^{7}$ John Rawls: A Theory of Justice, Oxford, 1971, p. 85.

${ }^{8}$ Carlos S. Nino: Ética y derechos humanos, Paidos, Buenos Aires, 1984, p. 241.
} 
sentido distinguir una justicia procesal pura perfecta de otra imperfecta.

Efectivamente, esta distinción serviría para resaltar nítidamente la diferencia que existe entre el discurso moral y la democracia, ya que mientras el primero sería un caso paradigmático de «justicia procesal pura perfecta» (pues sus reglas constitutivas son el único criterio de adecuación de los principios sustantivos que sirven como justificación última de cualquier institución, acción o actitud), la democracia constituye un supuesto de «justicia procesal pura imperfecta», en el que las desviaciones del modelo perfecto estarían impuestas por razones pragmáticas.

Esta clasificación de Rawls de los distintos tipos de justicia procesal, una vez introducidas las modificaciones de Nino -que aparecen en negrita- puede representarse como sigue:

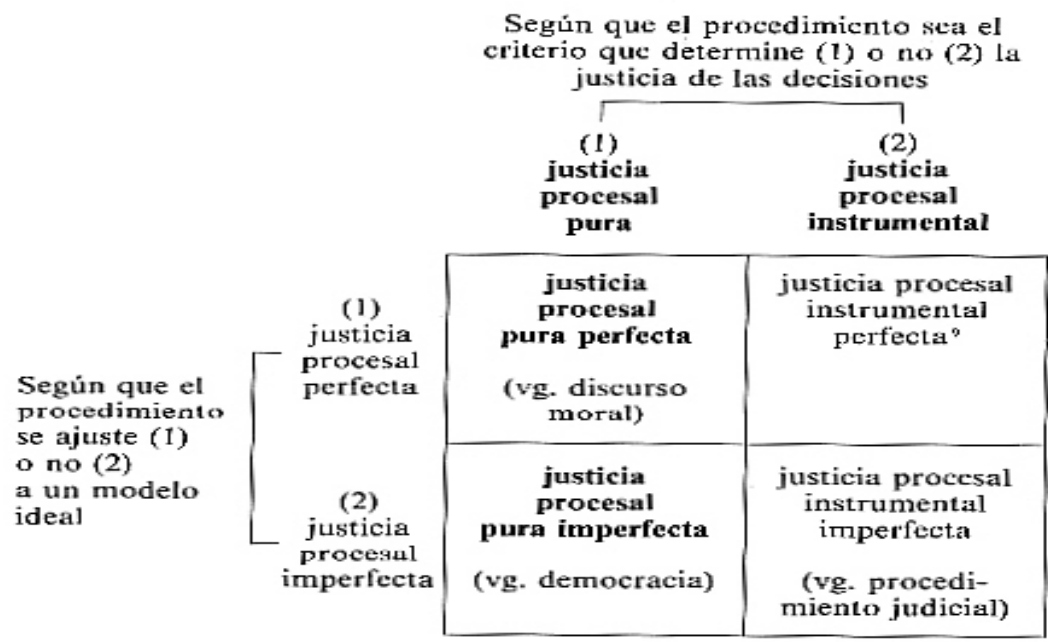

${ }^{9}$ Carlos S. Nino no cita ningún caso de «justicia procesal instrumental perfecta», pero Rawls menciona un supuesto de «justicia procesal perfecta» 
La doble consideración de la democracia como un supuesto no sólo de «justicia procesal pura» sino también de «justicia procesal imperfecta» trae consigo una serie de consecuencias respecto de las decisiones adoptadas de acuerdo a tal procedimiento. Así, las consecuencias ligadas a la primera consideración pueden desglosarse del siguiente modo:

a. la justicia de las decisiones democráticas viene determinada exclusivamente por el procedimiento que se siguió para adoptarlas;

b. para evaluar la justicia de las decisiones democráticas no existen criterios distintos al propio procedimiento;

c. la justicia de las decisiones democráticas no puede evaluarse en razón de su contenido.

Las consecuencias ligadas a la consideración de la democracia como un supuesto de «justicia procesal pura imperfecta» serían las siguientes:

a. al adoptar decisiones democráticas pueden darse desviaciones con respecto al procedimiento perfecto (el discurso moral) impuestas por razones pragmáticas;

b. las decisiones democráticas gozan sólo de una justificación «prima facie»;

c. la justificación de las decisiones democráticas queda supeditada a revisión exclusivamente a la luz de las desviaciones que se produzcan con respecto al procedimiento perfecto (el discurso moral).

que sirve para ilustrar aquella noción: «Un pastel habrá de dividirse entre un número de personas: suponiendo que una división justa sea una división igualitaria; ¿cuál es el procedimiento, si lo hay, que dará ese resultado? Haciendo a un lado los tecnicismos, la solución obvia es la de que una persona divida el pastel y tome la última parte, permitiendo a los otros que escojan antes. Dividirá el pastel en partes iguales, ya que de este modo estará seguro de obtener la mayor porción posible». Cfr. John Rawls, Teoría de la Justicia, Fondo de Cultura Económica, México, 1895, p. 108.

${ }^{10}$ Para un análisis del recurso a otros posibles criterios, tales como el consentimiento, el autointerés, las intuiciones morales o los presupuestos formales del razonamiento moral, puede verse: «Constructivismo espitemológico: entre Rawls y Habermas» de Carlos S. Nino, en Doxa. Cuadernos de Filosofía del Derecho, Alicante, núm. 5, 1988, pp. 87 a 105.

${ }^{11}$ Mientras que las anteriores consecuencias son comunes a los casos de «justicia procesal instrumental imperfecta», esta última se da únicamente en los supuestos «justicia procesal pura imperfecta». 
Como conclusión de lo dicho conviene resaltar aquí que Nino en esta primera edición de Ética y derechos humanos considera que el discurso democrático constituye un caso de «justicia procesal pura imperfecta», rechazando expresamente que pueda tratarse de un supuesto de «justicia instrumental imperfecta».

2.2. Posteriormente, en un artículo titulado «La paradoja de la irrelevancia moral del gobierno y el valor epistemológico de la democracia» ${ }^{12}$, Nino parece suavizar la tesis anterior negando expresamente que la democracia sea un caso de «justicia procesal pura imperfecta» y atribuyéndole simplemente un valor epistemológico.

Así, en la parte central de este artículo se plantea si la democracia posee un valor ontológico o únicamente epistemológico. Afirmar lo primero supone que a través del discurso democrático podemos constituir la verdad moral y que la democracia es un caso de «justicia procesal pura», aunque imperfecta:

«Mientras que el discurso moral es un supuesto de justicia procesal pura perfecta (para emplear, completándola, la distinción rawlsiana), ya que el único criterio de validez de sus resultados es que se hayan seguido sus reglas y éstas aseguran esa validez, la democracia es un procedimiento de justicia procesal pura pero imperfecta, porque sus reglas implican algún apartamiento del modelo ideal» ${ }^{13}$.

Por lo tanto, la validez de los resultados a los que se llega a través del discurso democrático «tendrá mayor o menor grado en la medida en la que haya un mayor o menor apartamiento de las reglas del discurso moral originario».

Pese a que esta caracterización del valor moral de la democracia es idéntica a la de la primera edición de Ética y derechos humanos, Nino prosigue su exposición preguntándose «si esta concepción ética es adecuada» y afirmando que «la pregunta despierta dudas fundadas».

En efecto, para Nino toda discusión real presupone la existencia de principios morales válidos que son el resultado de un consenso ideal:

12 Carlos S. Nino: «La paradoja de la irrelevancia moral del gobierno y el valor epistemológico de la democracia», Análisis Filosófico, Buenos Aires, vol. VI, núm. 2, 1986, pp. $75-82$.

${ }^{13}$ Ob. cit., p. 77. 
«¿Cuál es el criterio de validez que se presupone en el discurso o discusión moral? He sugerido en otro lugar que este criterio se basa en el hecho de la aceptabilidad hipotética del principio en cuestión, por todos los que puedan estar afectados por ese principio... en condiciones de racionalidad, imparcialidad y conocimientos plenos» ${ }^{14}$.

Siendo así las cosas, habría que descartar esta primera concepción que ve a los principios morales como el resultado de la discusión real. En cualquier caso, «lo que fracasa, aparentemente, es el constructivismo ontológico..., todavía podría defenderse el constructivismo epistemológico» que sostiene que la discusión favorece el acceso a la verdad moral ${ }^{15}$ :

«La participación en la discusión de quienes pueden estar afectados por la adopción de un principio u otro, sobre todo cuando tienen intereses opuestos, es la mejor garantía de que, si las reglas de la discusión fueron aceptadas, el consenso real obtenido se aproxima al consenso ideal. Esta garantía no se da en la reflexión individual o aislada o en una discusión limitada a unas pocas personas con intereses coincidentes»).

Finalmente, conviene señalar que la afirmación de un valor epistemológico de la democracia abre el camino para que las decisiones democráticas puedan ser cuestionadas no sólo por el grado en que se hayan satisfecho los requisitos de la discusión (lo que también sucedía afirmando su carácter ontológico), sino también, y fundamentalmente, recurriendo a criterios capaces de evaluar la justicia de los resultados con independencia del procedimiento.

La segunda edición de Ética y derechos humanos ${ }^{16}$ no contempla cambios sustanciales respecto de esta última tesis. Básicamente se limita a reafirmar el «valor epistemológico de la democracia» y desaparecen las alusiones expresas a cualquier tipo de justicia procesal. En todo caso, merece la pena resaltar, como luego se verá, que el principal argumento empleado en favor del carácter epistemológico de la democracia radica en su analogía con el proceso judicial, si bien se trata de «un procedimiento en el cual todos son parte y todos son jueces»».

${ }^{14}$ Ob. cit., p. 78

${ }^{15}$ Ob. cit., p. 79.

${ }^{16}$ Carlos S. Nino: Ética y derechos humanos. Un ensayo de fundamentación, (2. a edición), Astrea, Buenos Aires, 1989, pp. 393-398. 
2.3. De lo dicho hasta aquí por el Nino posterior a la primera edición de Ética y Derechos Humanos, dos afirmaciones en torno al valor de la democracia parecen claras: que carece de valor ontológico, y por tanto no es un caso de justicia procesal pura imperfecta, y que posee un valor epistemológico. Lo que cabe preguntarse ahora es dónde se sitúa la democracia dentro de los distintos tipos de justicia procesal que Nino propone. En este sentido, parece que la reconstrucción más plausible de la tesis de Nino lleva, como vamos a ver, a afirmar que se trata de un supuesto de «justicia procesal instrumental imperfecta».

Efectivamente, Nino parece usar como equivalentes las expresiones «constructivismo ontológico» y «justicia procesal pura». Así, en el primer caso el procedimiento es el criterio que determina los valores morales y en el segundo las decisiones justas. Por lo tanto, como hemos visto, la negación del constructivismo ontológico lleva a Nino a renunciar a la idea de que la democracia pueda ser un supuesto de «justicia procesal pura imperfecta».

Ahora bien, lo que cabe preguntarse en primer término es si afirmar el valor epistemológico de la democracia es también equivalente a considerarla como un supuesto de «justicia procesal instrumental». Parece que existen razones más que fundadas para ello, no sólo por la relación con la equivalencia constructivismo ontológico-justicia procesal pura, sino, fundamentalmente, porque tanto en el constructivismo epistemológico como en la «justicia procesal instrumental» el procedimiento, por sí mismo, no determina la justicia de los resultados. Esto hace no sólo posible, sino también conveniente, recurrir a criterios independientes del procedimiento para evaluar la justicia de los resultados (Vg. el consentimiento, el autointerés, las intuiciones morales, los presupuestos formales del razonamiento moral, etc.).

Estas relaciones de equivalencia entre los distintos tipos de constructivismo, por un lado, y de justicia procesal, por otro, pueden representarse como sigue: 


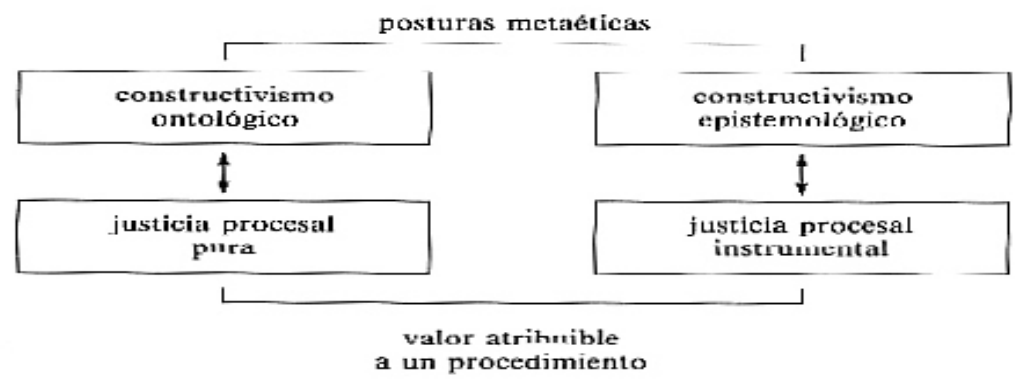

En segundo término cabe plantearse si la democracia constituye un caso de justicia procesal instrumental perfecta o imperfecta. Para responder adecuadamente a esta pregunta hay que comenzar observando que la oposición que Nino establece entre procedimiento perfecto e imperfecto corre paralela a la necesidad de distinguir entre el discurso moral y el discurso democrático.

Efectivamente, en la primera edición de Ética y derechos humanos, Nino introduce una distinción entre los «procedimientos de justicia procesal pura perfecta e imperfecta» basada en que en las reglas de éstos últimos se ha producido «algún apartamiento del modelo ideal». Así, como hemos visto, el discurso moral sería un procedimiento del primer tipo y el discurso democrático del segundo y la diferencia entre ambos radica en «el reemplazo de la exigencia del consenso unánime por el de la aprobación mayoritaria de una pauta o línea de acción».

Cuando más adelante Nino niega el valor ontológico del discurso moral se ve obligado también a negar el del discurso democrático y a dotar a ambos de un valor epistemológico. No obstante, continúa sosteniendo que los dos discursos no están en el mismo plano y que el segundo es un sucedáneo del primero. Parece claro, pues, que subsistiendo el mismo criterio de diferenciación entre ambos discursos, puede también trazarse la diferencia entre procedimiento perfecto e imperfecto dentro de los supuestos de justicia instrumental.

Otro argumento en favor de la consideración de la democracia como un caso imperfecto de justicia instrumental lo aporta la distinción que según Rawls existe entre la «justicia procesal perfecta» y la «justicia procesal imperfecta» (conviene recordar 
que para Nino ambos supuestos tienen en común su carácter instrumental). En este sentido, mientras que el primer caso «conduce infaliblemente a resultados considerados justos según criterios independientes», el segundo «tiende a producir, pero no asegura, resultados considerados justos según criterios independientes». No parece, por lo tanto, que sea inadecuado situar al discurso democrático en este segundo contexto.

En todo caso, valga el argumento adicional de que como ejemplo de justicia procesal instrumental imperfecta se cita en la primera edición de Ética y derechos humanos el procedimiento judicial y que en la segunda edición el discurso democrático se asimila a un procedimiento judicial en el cual todos son juez y parte.

La reconstrucción que propongo de la tesis del Nino de la segunda edición de Ética $y$ derechos humanos puede representarse como sigue:

\begin{tabular}{|c|c|c|}
\hline & $\begin{array}{c}\text { constructivismo } \\
\text { ontológico } \\
\downarrow \\
\text { justicia procesal } \\
\text { pura }\end{array}$ & $\begin{array}{c}\text { constructivismo } \\
\text { epistemológico } \\
\downarrow \\
\text { justicia procesal } \\
\text { instrumental }\end{array}$ \\
\hline $\begin{array}{l}\text { justicia } \\
\text { procesal } \\
\text { perfecta }\end{array}$ & $\begin{array}{c}\text { justicia procesal } \\
\text { pura } \\
\text { perfecta }\end{array}$ & $\begin{array}{c}\text { justicia procesal } \\
\text { instrumental } \\
\text { perfecta }\end{array}$ \\
\hline $\begin{array}{l}\text { justicia } \\
\text { procesal } \\
\text { imperfecta }\end{array}$ & $\begin{array}{c}\text { justicia procesal } \\
\text { pura } \\
\text { imperfecta }\end{array}$ & 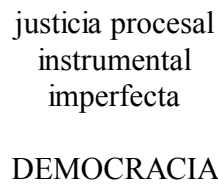 \\
\hline
\end{tabular}

Esta concepción de la democracia como un supuesto de «justicia procesal instrumental imperfecta» trae consigo una serie de consecuencias ligadas a la caracterización de la democracia como «instrumental», por un lado, y como «imperfecta», por otro. Las primeras pueden desglosarse como sigue:

a. el procedimiento democrático posibilita el conocimiento de decisiones justas;

b. la justicia de las decisiones democráticas no viene determinada por el procedimiento que se siguió para adoptarlas; 
c. para evaluar la justicia de las decisiones democráticas puede recurrirse a criterios distintos del procedimiento (consenso hipotético, autointerés, intuiciones morales, presupuestos formales del razonamiento moral, etc);

d. la justicia de las decisiones democráticas puede evaluarse en razón de su contenido.

Las consecuencias que acarrea la consideración de la democracia como «imperfecta» pueden expresarse como sigue:

a. al adoptar decisiones democráticas pueden darse desviaciones con respecto al procedimiento perfecto (el discurso moral) impuestas por razones pragmáticas;

b. las decisiones democráticas gozan sólo de una justificación «prima facie»;

c. las desviaciones respecto del procedimiento perfecto pueden ocasionar decisiones consideradas injustas (en virtud de criterios independientes).

3. A partir de las consecuencias que se extraen de esta nueva caracterización de la democracia como un supuesto de «justicia procesal instrumental imperfecta» pueden formularse una serie de conclusiones, centrándonos en los límites de este tipo de justificación y dejando a un lado el carácter más o menos artificioso de la clasificación de los diferentes tipos de justicia procesal, así como los problemas del cambio en la caracterización del propio discurso moral:

3.1. En primer lugar, se aprecia un debilitamiento de la fuerza justificatoria del procedimiento democrático sobre las decisiones que se adoptan con arreglo al mismo. De esta manera, en la primera caracterización de la democracia que hemos visto -como «justicia procesal pura imperfecta»- las decisiones democráticas quedan justificadas en tanto que son el producto de un procedimiento democrático; basta con que se den una serie de criterios formales para considerar que las decisiones son justas, sin necesidad de analizar su contenido. En cambio, en la caracterización de la democracia que ahora nos ocupa, la justicia de las decisiones democráticas viene dada por criterios ajenos al procedimiento; lo que determina la justicia de tales decisiones es la conformidad o no de sus contenidos con algún criterio diferente del procedimiento democrático, si bien éste sigue siendo un método más o menos fiable para conocer decisiones justas. 
3.2. En segundo lugar, se produce un debilitamiento correlativo al anterior en la propia justificación del procedimiento democrático. De manera que en la primitiva caracterización de la democracia ésta quedaba fuertemente justificada al tratarse de un procedimiento para constituir decisiones justas, mientras que ahora, es sólo un procedimiento para conocer decisiones justas pues - como hemos visto- el procedimiento, de por sí, no determina la justicia de la decisión.

Pero es que además la democracia, tal y como queda caracterizada ahora, no constituye ni tan siquiera un criterio excluyente para el conocimiento de la verdad moral, siendo posible recurrir a otros criterios para ello. Pues aún considerando, como hace Nino, que el único criterio de validez aceptable es la adecuación a los presupuestos formales del razonamiento moral (que constituyen la verdad moral) ${ }^{17}$, todavía cabe una reflexión en torno a la verdad moral que se rija por reglas diferentes a las del discurso democrático. Y sobre todo, como el propio Nino admite, es posible, aunque difícil, que por vía de la reflexión individual se llegue a conclusiones correctas ${ }^{18}$.

3.3. Finalmente, conviene advertir que la nota de la «imperfección»-que parece persistir en esta nueva caracterización de la democracia- puede predicarse de la democracia no sólo cuando se la entiende como «modelo ideal», sino también, obviamente, aún más cuando nos referimos a la democracia real o representativa. Así, en el modelo ideal de democracia que configura Nino subsisten todos los rasgos del discurso moral originario excepto aquellos que hacen que ese discurso sea un método inestable e inconcluyente para alcanzar decisiones colectivas; de esta manera, el reemplazo de la exigencia de consenso unánime por el de aprobación mayoritaria de las decisiones determina la calificación del procedimiento democrático como «imperfecto».

Pero también cuando nos referimos a la democracia real o representativa tiene sentido hablar de la imperfección de dicho procedimiento. La democracia representativa es un sucedáneo imperfecto de la democracia ideal (así como ésta lo es del discurso moral), ya que las exigencias que configuran el modelo democrático ideal de Nino (tales como las de que debe tratarse que las decisiones estén justificadas sobre la base de principios que satisfagan las condiciones formales del discurso moral, que para

${ }^{17}$ Carlos S. Nino: «Constructivismo epistemológico: Entre Rawls y Habermas», Doxa. Cuadernos de Filosofía del Derecho, núm. 5, 1988, pp. 87 a 105.

${ }^{18}$ Ob. cit., p. 104. 
ello deben escucharse los argumentos a favor o en contra de que esta satisfacción se da, que toda persona moral debe considerarse una fuente potencial de tales argumentos, que todas ellas deben participar en las decisiones colectivas, etc. ${ }^{19}$ ) tienen tendencia a desdibujarse o desaparecer en la mayor parte de los sistemas democráticos reales que conocemos. Y es precisamente el grado con que esta imperfección se plantea ${ }^{20}$, lo que puede hacer inviable una justificación de la democracia derivada de un modelo ideal como el que Nino propone.

${ }^{19}$ Carlos S. Nino: Ética y derechos humanos. Un ensayo de fundamentación (2. a edición), Astrea, Buenos Aires, 1989, p. 392.

${ }^{20}$ Para un detallado análisis de este tema puede verse: Juan Ramón Capella, Entre sueños. Ensayos de filosofía política, Icaria, Barcelona, 1985.

\section{DOXA-10 (1991)}

